УДК 51-7

DOI: $10.18101 / 2304-5728-2019-1-78-90$

\title{
ОБ ОПТИМИЗАЦИИ СЕТЕЙ ИНЖЕНЕРНЫХ КОММУНИКАЦИЙ РАЗЛИЧНОГО НАЗНАЧЕНИЯ ${ }^{1}$
}

\section{(C) Токтошов Гулжигит Ысакович}

кандидат технических наук, научный сотрудник, Институт вычислительной математики и математической геофизики СО РАН Россия, 630090, г. Новосибирск, пр-т Академика Лаврентьева, 6 E-mail: tgi_tok@rambler.ru

\section{(C) Мигов Денис Александрович}

кандидат физико-математических наук, старший научный сотрудник, Институт вычислительной математики и математической геофизики СО РАН Россия, 630090, г. Новосибирск, пр-т Академика Лаврентьева, 6 E-mail:mdinka@rav.sscc.ru

Рассматриваются задачи оптимизации сетей инженерных коммуникаций по критерию минимума суммарных строительных затрат при условии, что проектируемая сеть должна также удовлетворять заданному порогу надежности. Предполагается, что сбои происходят в каналах связи первичной сети, при этом узлы во вторичной сети должны быть связаны достаточно надежно. В качестве показателей надежности рассмотрены три характеристики. Предлагается новая методика моделирования сетей инженерных коммуникаций, позволяющая, в отличие от классических моделей, учитывать, во-первых, иерархичность структуры проектируемой сети и, во-вторых, совместимость проектируемой сети с другими, уже существующими или вновь проектируемыми сетями различного назначения. В итоге получен алгоритм построения сетей, который может быть применен при модернизации существующих, либо проектировании новых сетей в соответствии с планом развития и расширения современных городов. Предложенная методика построения сетей различного назначения учитывает уже на этапе проектирования особенности проектируемых типов сетей, а также их иерархичность. Кроме того, приближенный алгоритм, предложенный в настоящей работе, позволяет построить наиболее дешевый сетевой объект с учетом его надежности.

Ключевые слова: область размещения; целевая продукция; потребители; линейное сооружение; инженерная сеть; трасса; первичная сеть; вторичная сеть; граф; гиперсеть; надежность; эвристика; альтернативный маршрут.

\footnotetext{
${ }^{1}$ Работа поддержана Программой фундаментальных исследований ИВМиМГ СО РАН №0315-2016-0006 и проектами РФФИ № 18-07-00460, 17-07-00775.
} 
Г. Ы. Токтошов, Д. А. Мигов. Об оптимизации сетей инженерных коммуникаций различного назначения

Для цитирования:

Токтошов Г. Ы., Мигов Д. А. Об оптимизации сетей инженерных коммуникаций различного назначения // Вестник Бурятского государственного университета. Математика, информатика. 2019. № 1. С. 78-90.

\section{Введение}

Задачи проектирования сетей инженерных коммуникаций различного назначения (газо-, водопроводы, теплосети, сети связи и электроснабжения и т. п.) в условиях городской застройки возникают при строительстве новых или модернизации существующих сетей с целью обеспечения потребности населенных пунктов, торговых площадок, центров обслуживания заявок и т. п.

Известные постановки задачи оптимизации сетей и подходы к их решению основываются на общих принципах построения и эксплуатации этих сетей. В частности, для решения сложных проектных задач, заключающихся в анализе и синтезе систем сетевой структуры, используется подход разделения задачи на структурную и параметрическую оптимизации. Зачастую процесс анализа связан с инженерным изысканием и предварительной подготовкой исходных данных.

При решении задач структурной оптимизации сетевых структур различного назначения, авторы [1-3] используют методологию избыточных проектных схем или граф всех возможных трасс [4].

В целом избыточная схема (ИС) - эта схема, которая должна учитывать все ограничения на плане местности, включая места расположения источников целевой продукции (информация, энергия, продукт) и потребителей, а также всевозможные (допустимые с точки зрения технической возможности и экономической целесообразности) связи между ними. Тогда задачу структурной оптимизации сетей инженерных коммуникаций по критерию минимума приведенных затрат и их функционирования в общем виде можно сформулировать как выделение подсети на заданной избыточной схеме, обеспечивающей минимум затрат на ее строительство и эксплуатацию. Если необходимо обеспечить также надежное функционирование проектируемой инженерной сети, к сформулированной задаче добавляется требование о том, чтобы надежность сети не была меньше чем наперед заданное пороговое значение. В этом случае возможно использование различных показателей надежности. Далее мы вводим в рассмотрение и описываем некоторые из них.

\section{1 Методологический подход к моделированию сетей}

Отметим, постановка задачи структурной оптимизации инженерных сетей сразу по двум критериям является уже новой, не поддающей к ращению в общем случае. Подобные задачи в рамках классических подходов обычно решаются на одной плоскости, т. е. задача сводится к тому, 
чтобы выделить подсеть из заданной избыточной схемы, удовлетворяющей заданному критерию. У такого подхода есть одно бесспорное преимущество, связанное с доступностью проведения вычислительного эксперимента на основе классических схем оптимизации, таких как методы имитации отжига [2], методы теории графов [3; 4] и другие.

Однако следует отметить, что при решении подобных задач необходимо обратить внимание и на другую сторону этого вопроса, связанного со следующими особенностями прикладной проблемы:

- во-первых, решение нужно искать не на плоскости (как это делается, например, в работах [1-4]), а в пространстве [5], учитывающем взаимодействие двух объектов (иерархичность сетей), таких как область размещения со своими технико-экономическими характеристиками и проектируемая сеть, имеющая также свои технико-экономические характеристики в зависимости от выбранной местности;

- во-вторых, при проектировании любой системы необходимо учесть тот факт, что любая сеть взаимодействует (сосуществует) с сетями различного назначения (уже существующими или вновь проектируемыми).

\section{2 Математическая модель}

Согласно первой особенности проектируемой сети, ее структура представляет собой четырехуровневую взаимосвязанную систему: участок земли $\rightarrow$ система фундаментов или опор $\rightarrow$ технологическая труба $\rightarrow$ целевая продукция. В таком случае применение аппарата теории графов и гиперграфов не позволяет учесть реализацию одной из представленных систем в другой. Поэтому в большинстве случаев вместо них используют другие сетевые модели: вложенные графы [6], многоуровневые комплексные сети [7], гиперсети [8], а также другие объекты.

Приведем наиболее общее определение гиперсети [8], применяемой в нашем случае.

Определение. Гиперсеть $H N=(X, V, R ; P, F, W)$ включает следующие объекты:

$X=\left(x_{1}, x_{2}, \ldots, x_{n}\right)-$ множество узлов (вершин);

$V=\left(v_{1}, v_{2}, \ldots, v_{g}\right)$ - множество возможных трасс;

$R=\left(r_{1}, r_{2}, \ldots, r_{m}\right)$ - множество линейных сооружений;

$P: V \rightarrow X^{2}$ - отображение, сопоставляющее каждому элементу $v \in V$ множество вершин $P(v) \subseteq X$. Тем самым отображение $P$ определяет граф первичной сети $P N=(X, V)$.

$F: R \rightarrow 2^{V}$ - отображение, сопоставляющее каждому элементу $r \in R$ множество трасс $F(r)$, образующих простой маршрут в графе $P N=(X, V)$. Множество всех маршрутов $F(r)$, отображающее каждо- 
Г. Ы. Токтошов, Д. А. Мигов. Об оптимизации сетей инженерных коммуникаций различного назначения

му ребру $r \in R$ графа $S N$ единственный маршрут в графе $P N$, назовем вложением графа $S N$ в $P N$.

$W: R \rightarrow 2^{P(F(R))}$ - отображение, сопоставляющее каждому элементу $r \in R \quad$ множество $\quad$ вершин $\quad W(r) \subseteq P(F(r)) \quad$ в $\quad$ графе $P N, \quad$ где $P(F(r))=Y-$ множество вершин в $P N$, инцидентных трассам $F(r) \subseteq V$. Отображение $W$ определяет граф вторичной сети $S N=(Y, R)$.

Из определения гиперсети следует, что математическая модель возможных трасс соответствует графу первичной сети $P N=(X, V)$, а структура инженерной сети — графу вторичной сети $S N=(Y, R)$. Взаимодействие этих сетей определяется гиперсетью $H N$.

Важно также учесть возможность совмещенной прокладки параллельно идущих сетей в одном техническом коридоре. При этом необходимо учесть совместимость или несовместимость этих сетей и нормативное расстояние между ними [9].

\section{3 Надежность гиперсетей}

Отметим, что анализ надежности гиперсетей впервые предоставлен в $[10 ; 11] . \quad$ В качестве меры надежности гиперсетей рассматривают 2-терминальную надежность, т. е. вероятность связности двух выделенных узлов сети (терминалов) при условии, что в первичной сети происходят сбои, а узлы во вторичной сети должны быть надежными.

Будем считать, что ветви $V$ первичной $P N=(X, V)$ сети подвержены случайным отказам, происходящим независимо друг от друга с заданными вероятностями $p_{i}, 1 \leq i \leq g$.

Надежность ребра вторичной сети $r \in R$ определим как

$$
R_{r}(H N)=\prod_{v \in F(r)} p(v) \text {. }
$$

Если для ребра $r \in R$ путь $F(r)$ имеет конечные точки $a$ и $b$ и никакие другие ребра не связывают эти точки, то мы будем использовать обозначение $R_{a b}(H N)$ вместо $R_{r}(H N)$. Если для узлов $a$ и $b$ существует более одного такого ребра, это обозначение используется для того ребра, значение надежности которого максимально.

Ранее в $[12 ; 13]$ мы рассматривали меру надежности $R(H N)$ для гиперсети $H N$ в целом с учетом того, что в первичной сети происходят сбои, но при этом все потребители должны быть связаны с необходимыми им поставщиками:

$$
R_{1}(H N)=\min \left\{R_{a b}(H N)\right\}, a \in Y_{\text {source }}, b \in Y_{\text {consumer }} .
$$


Таким образом, $R_{1}(H N)$ является минимумом среди всех 2-терминальных надежностей $R_{a b}(H N)$, где $a-$ это источник ресурса, $b$ - потребитель.

Однако не во всех случаях адекватной мерой надежности будет именно указанный показатель. Введем в рассмотрение показатель для описания надежность сети в среднем:

$$
R_{2}(H N)=\frac{1}{\Omega} \sum_{1}^{\Omega} R_{a b}(H N), a \in Y_{\text {source }}, b \in Y_{\text {consumer }},
$$

где $\Omega$ - количество всех рассматриваемых пар источник - потребитель.

Рассмотрим также показатель, который необходим для описания надежности в случае, когда нужно чтобы все потребители и поставщики одновременно были связны с максимальной вероятностью. Определим его как вероятность существования путей между каждой парой источник потребитель:

$$
R_{3}(H N)=\operatorname{probability}\left(\text { Exist_a_path_between } a \in Y_{\text {source }}, b \in Y_{\text {consumer }}\right) \text {. }
$$

Возможны ситуации, когда при одних и тех же условиях разные конфигурации инженерной сети будут оптимальными с точки зрения введенных показателей.

Надежность гиперсети $H N$ требует, чтобы были известны достаточно надежные маршруты между заданными парами точек в графе первичной сети $P S$.

$$
\begin{gathered}
4 \text { Постановка задачи } \\
D=\{(x, y) \mid a<x<b, c<y<d\} \text { - прямоугольная картографируемая }
\end{gathered}
$$
область, на которой предполагается строительство сетей различного назначения;

$Y_{\text {source }} \subseteq D-$ множество источников ресурсов (газ, нефть, вода и т.д.);

$Y_{\text {consumer }} \subseteq D-$ множество потребителей.

Множество вершин $X$ графа первичной сети $P N=(X, V)$ включает в себя множество $Y=Y_{\text {source }} \cup Y_{\text {consumer }}$.

Пусть в графе $P N=(X, V)$ заданы следующие характеристики:

$l(v)$ - длина ветви $v \in V$;

$a(v)$ - стоимость земли (аренда, налог и т. д.) на участке $v \in V$ предполагаемой трассы;

$b(v)$ - стоимость подготовительных и строительных работ (копка траншеи, строительство коллекторов, тоннелей и опор и т. п.) на участке $v \in V$ предполагаемой трассы; 
Г. Ы. Токтошов, Д. А. Мигов. Об оптимизации сетей инженерных коммуникаций различного назначения

$\gamma_{1}$ - коэффициент дисконтирования строительных затрат для приведения экономических показателей разных лет к сопоставимым по времени величинам.

Пусть в графе вторичной сети $S N=(Y, R)$ заданы следующие параметры:

$$
\rho(r)=\sum_{v \in F(r)} l(v) \text { - длина линейного сооружения (трубопровод, ка- }
$$

бель и т. п.) $r \in R$, проходящего по ветвям $v \in F(r)$ предполагаемой трассы;

$c(r)$ - стоимость строительства и монтажа самого линейного сооружения $r \in R$ соединяющего соответствующие пары узлов из $Y$;

$\gamma_{2}$ - коэффициент дисконтирования стоимости линейного сооружения;

$d_{v}(r)$ - стоимость эксплуатации сетей на участках $v \in F(r)$;

Пусть $T$ - множество типов сетей. Для каждого ребра $r \in R$ зададим тип type $(r) \in T$, который однозначно определяет вид и назначения проектируемой сети. Для описания совместимости различных типов сетей используем методику, предложенную в [12; 13]. Для этого вводится бинарное отношение $C T \subseteq T \times T$, которое определяется правилом: если $\left(t_{1}, t_{2}\right) \in C T$, то эти типы сетей могут быть проложены в одном и том же техническом коридоре, т. е. две различные, но совместимые (по механическим и электромагнитным воздействиям) сети могут быть проложены в одной ветви первичной сети.

Пусть $\operatorname{Min} C T\left(t_{1}, t_{2}, \ldots, t_{h}\right)$ - минимальное число непересекающихся подмножеств, на которое можно разделить типы сетей $\left\{t_{1}, t_{2}, \ldots, t_{h}\right\}$ с учетом их совместимости.

Например, если существует типы сетей $\left\{t_{1}, t_{2}, t_{3}\right\}$, такие что $\left(t_{1}, t_{2}\right),\left(t_{2}, t_{3}\right) \in C T$, но $\left(t_{2}, t_{3}\right) \notin C T$, тогда $\operatorname{Min} C T\left(t_{1}, t_{2}, t_{3}\right)=2$, поскольку эти типы сетей могут быть разделены на два подмножества $\left\{t_{1}, t_{2}\right\}$ и $\left\{t_{3}\right\}$.

Постановка задачи: построить гиперсеть $H N$, т. е. каждому ребру $r \in R$ графа вторичной сети $S N$ найти маршрут в графе первичной сети $P N$ между заданных пар точек из $Y=Y_{\text {source }} \cup Y_{\text {consumer }}$, для которой следующая целевая функция принимает минимальное значение: 


$$
\begin{aligned}
& Q(H N)=\sum_{v \in V^{\prime}}\left(a(v)+b(v) \cdot \gamma_{1}\right) \cdot \rho(v) \cdot \operatorname{Min} C T(v)+ \\
& +\sum_{r \in R}\left(c(r)+\sum_{v \in F(r)} d(r) \cdot \gamma_{2}\right) \rho(r)
\end{aligned}
$$

и обеспечивает надежность гиперсети по отношению к заданному порогу $R(H N) \geq R_{0}$, где $0<R_{0} \leq 1$.

В (1) подмножество $V^{\prime} \subseteq V$ определяется следующим образом: для каждого элемента $v \in V^{\prime}, \exists r \in R$ такое, что $v \in F(r)$. Пусть $v \in V^{\prime}$ и $v \in F\left(r_{i}\right), i=1, \ldots, l ; r_{1}, \ldots, r_{l} \in R$, тогда

$$
\operatorname{MinCT}(v)=\operatorname{MinCT}\left(\operatorname{type}\left(r_{1}\right), \ldots, \text { type }\left(r_{l}\right)\right) \text {. }
$$

Отметим, что задача оптимизации сетей инженерных коммуникаций в такой гиперсетевой постановке NP-трудна [14; 15].

В связи с этим для решения поставленной задачи предложен приближенный метод, основанный на следующих предпосылках. Во-первых, найти оптимум для отображения ребер графа $S N$ вторичной сети по соответствующим маршрутам на графе $P S$ первичной сети практически нереально в силу сложности задачи, поэтому достаточно найти удовлетворительное приближение [16]. Во-вторых, применение различных эвристик для улучшения первичного приближения, основанных на классических методах оптимизации. В качестве такой эвристики в настоящей работе был предложен метод, основанный на формировании множества доступных альтернативных маршрутов между узлами $\left(y_{i}, y_{j}\right), i, j=1, \ldots n, i \neq j$ графа $P S$ первичной сети для отображения ребер $r_{i} \in R$ графа $S N$ вторичной сети с учетом совместимости и надежности проектируемой сети.

Далее мы приведем способ построения доступных альтернативных маршрутов и метод для решения задач совмещенного размещения сетей различного назначения по критерию минимума строительных затрат при ограничениях на надежность будущей сети.

\section{5 Построение доступных альтернативных маршрутов для проектирования гиперсети}

Часто бывает необходимо иметь возможность определять альтернативные по стоимости маршруты в графе первичной сети $P S$, если какаялибо часть самого дешевого маршрута повреждена или занята другими видами вторичной сети $S N$. Для этого алгоритмом Йена [17] находим $k$-кратчайших путей, представляющих собой упорядоченный список доступных альтернативных маршрутов. 
Г. Ы. Токтошов, Д. А. Мигов. Об оптимизации сетей инженерных коммуникаций различного назначения

При этом самый кратчайший путь в графе первичной сети $P S$ между узлами $\left(y_{i}, y_{j}\right), i, j=1, \ldots n, i \neq j$ для отображения ребер $r_{i} \in R$ графа вторичной сети $S N$ является предпочтительным маршрутом. Если по какойто причине самый кратчайший путь между двумя узлами недоступен для отображения ребра $r_{i} \in R$, т. е. занят несовместимыми типами сетей или не удовлетворяет заданному порогу надежности, то необходимо определить второй кратчайший путь между $\left(y_{i}, y_{j}\right), i, j=1, \ldots n, i \neq j$. Если этот тоже недоступен, может понадобиться третий кратчайший путь. Полученную таким образом серию путей назовем как $k$-кратчайшими путями в графе первичной сети $P S$ для отображения ребер $r_{i} \in R$ вторичной сети $S N$. Из таких путей выбираются те маршруты, при которых стоимость гиперсети была бы минимальной, а ее надежность не была бы меньше чем наперед заданное пороговое значение. Известно, что алгоритм Йена является эффективным и широко используемым алгоритмом для определения $k$-кратчайших маршрутов. Отметим, что этот способ построения доступных альтернативных маршрутов используется на этапе улучшения первичного приближения, представленного в следующем алгоритме.

\section{6 Приближенный метод решения задачи}

В данном разделе предлагается приближенный алгоритм решения поставленной задачи, основанный на идеи двухэтапного метода, представленного в [16]. Сначала находится начальное приближенное решение, затем оно улучшается.

Для поиска начального решения используется концепция «жадного» алгоритма, основанная на классическом алгоритме Флойда. Для простоты будем считать $\forall r \in R: c(r)+d(r) \cdot \gamma_{2}=$ const .

Далее будут описаны принцип работы данного метода.

Этап 1 (построение первичного приближения):

Шаг 1. Разделить типы сетей на непересекающиеся подмножества $\operatorname{Min} C T$.

Шаг 2. Выбрать из $\operatorname{MinCT}$ подмножество совместимых типов $T^{\prime}$ с наибольшим количеством ребер (для которых еще не найдено вложение в первичную сеть).

Шаг 3. Найти все кратчайшие пути $\left(y_{i}, y_{j}\right), i, j=1, \ldots, n, i \neq j$ (существует ребро $r)$ в графе первичной сети $P N=(X, V)$ с помощью алгоритма Флойда. Паре вершин $\left(y_{i}, y_{j}\right)$ соответствует ребро $r$ во вторичной сети и tуре $(r) \in T^{\prime}$.

Выбрать среди них путь минимальной стоимостью. 
Осуществить вложение выбранного ребра $r$ по кратчайшему пути $\left(y_{i}, y_{j}\right)$ в графе $P N$.

Для всех $v \in F(r)$ в графе первичной сети $P N a(v):=0, b(v):=0$ (стоимость ветви равна нулю для прокладки остальных ребер из $T^{\prime}$ ).

Повторить Шаг 3 пока для всех ребер $r \in R$ вторичной сети, таких что type $(r) \in T^{\prime}$, не будут найдены и реализованы кратчайшие пути.

Шаг 4. Присвоить изначальные значения $a(v)$ и $b(v)$, которые обнулены на предыдущем шаге. Если $\operatorname{Min} C T \neq 0$, переходим на Шаг 2.

Отметим, что «жадный» алгоритм является приближенным, поэтому полученные решения не всегда оптимальны. Можно улучшить решение, найдя новый маршрут для каждого $r \in R$. Например, новый маршрут $F(r)$ дешевле для выбранного $r \in R$, если он включает в себя большее количество ветвей $v \in F(r)$ с нулевой стоимостью (ветви, которые уже используется для других ребер $r \in R$ ).

Можно упорядочить ребра по некоторому правилу, например, по числу наиболее редко используемых ветвей, либо специально введенной метрике, как и в [16]. Однако в среднем полученные результаты сильно не зависят от используемого метода упорядочения.

Этап 2 (улучшение первичного приближения):

Шаг 1. Упорядочить $r \in R$ по убыванию их веса (получим список $\left.\left\{r_{i}\right\}\right)$.

Шаг 2. «Удалить» $r_{i}$ из гиперсети $H N$ согласно списку, т. е. $\forall v_{k} \in F\left(r_{i}\right)$, восстановить начальные значения $a\left(v_{k}\right)$ и $b\left(v_{k}\right)$, если $v_{k}$ не входит в другие ребра.

Алгоритмом Йена [17] между конечными узлами $\left(y_{i}, y_{j}\right), i, j=1, \ldots n, i \neq j \quad$ выбранного ребра $r_{i} \in R$ найти все $k$-кратчайшие пути, представляющие упорядоченный список доступных альтернативных маршрутов в графе $P N$.

Для выбранного ребра $r_{i} \in R$ и $l$-го кратчайшего маршрута для него, где $1 \leq l \leq k$, проверить: если $l$-й кратчайший маршрут удовлетворяет условию совместимости типов сетей, то $r_{i} \in R$ укладываем по $l$-му кратчайшему маршруту, в противном случае переходим к $l+1$-му кратчайшему маршруту и т. д. При этом для каждого $r_{i} \in R$ одновременно проверяется выполнения условия надежности для каждого рассматриваемого $l$-го кратчайшего маршрута: $R(l)>R_{0}$, где $0<R_{0} \leq 1$.

Повторить Шаг 2 пока все ребра $\left\{r_{i}\right\}$ из списка не будут обновлены.

Можно использовать несколько итераций этапа 2 для поиска построения наиболее подходящей структуры. 
Г. Ы. Токтошов, Д. А. Мигов. Об оптимизации сетей инженерных коммуникаций различного назначения

Предложенный двухэтапный эвристический метод, в отличие от метода, предложенного в [16], является более универсальным. Он учитывает возможность рассмотрения всех $k$-кратчайших путей, которые приводят к построению наиболее дешевого, в то же время достаточно надежного решения с учетом совместимости различных типов коммуникаций в одном техническом коридоре.

Отметим также, что $k$ в данном случае является важнейшим параметром алгоритма. Так, если $k$ достаточно мал, то на определенном этапе может оказаться, что все найденные $k$-кратчайшие пути не будут удовлетворять условиям совместимости либо надежности. В этом случае есть две стратегии: нахождение $k+1$-го пути (и далее $k+2$-го и т. д.) либо выбор их имеющихся самого надежного с выполнением условия совместимости. В первом случае получаем алгоритм существенно большей трудоемкости, но при этом значительно выше вероятность найти удовлетворительное решение. Второй вариант - компромиссный, его имеет смысл использовать, когда сложно адекватно спрогнозировать требуемую меру надежности и, возможно, довольствоваться просто самым надежным решением из приемлемых.

\section{Заключение}

В настоящей работе приведена постановка задач оптимизации сетей по критерию минимума приведенных затрат на строительство и эксплуатацию при ограничениях на надежность с учетом совместимости различных типов коммуникаций для прокладки в одном техническом коридоре. Предложен приближенный алгоритм поиска решения, основанный на модели гиперсети и идеи двухэтапного метода [16]. В отличие от базового двухэтапного алгоритма предлагаемый модифицированный вариант позволяет построение решения, находя целесообразный вариант решений среди $k$-кратчайших путей. Среди таких путей рассматривается возможность выбора маршрута, приводящего к компромиссному решению достаточно дешевому, и в то же время учитывающему совместимость различных типов коммуникаций и достаточно надежному решению. Так как не существует однозначного выбора такого маршрута, то его выбор осуществляется эвристическими подходами, основанными на моделях теории графов и теории гиперсетей.

Предложенная методика построения сетей различного назначения учитывает уже на этапе проектирования совместимость или несовместимость проектируемых типов сетей, а также их иерархичность. 


\section{Литература}

1. Соловьев А. Ю. Алгоритмы структурной оптимизации и методы анализа информационных процессов в современных телекоммуникационных системах: автореф. дис. ... канд. техн. наук. Воронеж, 2011. 18 с.

2. Стенников В. А., Чемезов А. А. Применение алгоритма перебора деревьев и метода имитации отжига для схемно-структурной оптимизации тепловых сетей // Программные продукты и системы. 2018. № 2 (31). С. 387-395.

3. Наумов И. В., Ямщикова И. В. Математическое обоснование выбора оптимизационной модели трассировки электрической сети // Евразийский союз ученых (ЕСУ). 2015. № 7 (16). С. 123-127.

4. Степанов В. П. Оптимизация маршрутов на дорожной сети // Наука и образование. 2012. №5. С. 1-12.

5. Попков В. К., Токтошов Г. Ы. Гиперсетевая технология оптимизации инженерных сетей в горной или пересеченной местности // Вестн. Бурят. гос. ун-та. Сер. Математика и информатика. 2010. Вып. 9. С. 276-282.

6. Poulovassilis A., Levene M. A Nested-Graph Model for the Representation and Manipulation of Complex Objects // J. ACM Trans. Inf. Syst. 1994. Vol.12. P. 35-68.

7. Kurant M., Thiran P. Layered Complex Networks // J. Phys. Rev. Lett. 2006. Vol. 96. P. 1-4.

8. Попков В. К. О моделировании городских транспортных систем гиперсетями // Автоматика и телемеханика. 2011. Т. 72, № 6. С. 179-189.

9. Автоматизированное проектирование генерального плана сооружений биохимической очистки сточных вод [Электронный pecypc]. URL: http://www.gaps.tstu.ru/win-1251/lab/gis/genplan.html (дата обращения: 19.10.2018).

10. Rodionov A. S., Rodionova O. K. Random Hypernets in Reliability Analysis of Multilayer Networks // J. Lecture Notes in Electrical Engineering. 2015. Vol. 343. P. 307-315.

11. Rodionov A. S., Rodionova O. K. Using Random Hypernets for Reliability Analysis of Multilevel Networks // 1st Int. Conf. on Mathematical Methods and Computational Techniques in Science and Engineering (MMCTSE 2014). Ser. Mathematical Methods in Science and Engineering. Greece, Athens, 2014. P. 119-121.

12. Toktoshov G., Yurgenson A., Migov D. Design of Utility Network Subject to Reliability Constraint // Proc. of International Multi-Conference on Engineering, Computer and Information Sciences, IEEE SIBIRCON. Novosibirsk, 2017 (18-22 Sept. 2017). P. 172.

13. Toktoshov G. Y., Yurgenson A. N., Migov D. A. On a Problem of the Utility Network Design // OPTA-SCL 2018. Springer, 2018. Vol. 2098. Р. 385-395.

14. Попков В. К. Трудно решаемые задачи теории гиперсетей // Дискретная оптимизация и исследование операций: материалы рос. конф. Новосибирск, 2007. C. 69-73.

15. Colbourn, Ch. J. The Combinatorics of Network Reliability. Oxford University Press, N. Y., 1987. P. 143-157.

16. Попков В. К., Токтошов Г. Ы., Юргенсон А. Н. Об одном подходе к оптимизации инфраструктуры инженерных сетей // Вестник СибГУТИ. 2012. № 3. C. 11-28.

17. Кристофидес Н. Теория графов. Алгоритмический подход. М.: Мир, 1978. $432 \mathrm{c}$. 
Г. Ы. Токтошов, Д. А. Мигов. Об оптимизации сетей инженерных коммуникаций различного назначения

ON THE OPTIMIZATION OF MISCELLANEOUS UTILITY NETWORKS

Gulzhigit Y. Toktoshov

Cand. Sci. (Engineering), Researcher, Institute of Computational Mathematics and Mathematical Geophysics SB RAS

6 Akademika Lavrentyeva Prospect, Novosibirsk 630090, Russia

E-mail: tgi_tok@rambler.ru

Denis A. Migov

Cand. Sci. (Phys. and Math.), Senior Researcher,

Institute of Computational Mathematics and Mathematical Geophysics SB RAS

6 Akademika Lavrentyeva Prospect, Novosibirsk 630090, Russia

E-mail: mdinka@rav.sscc.ru

The article deals with the problem of utility network optimization according to the criterion of the total construction cost minimum. In addition, we take into account the reliability constraints, so the future network must also meet preset reliability threshold. It is assumed that failures occur in communication channels of the primary network, and nodes in the secondary network should be connected reliably enough. Three characteristics are considered as indicators of reliability. We have proposed a new methodology for modeling utility networks, which unlike classical models allows taking into account the hierarchy of the future network, and its compatibility with other existing or newly developed miscellaneous networks. As a result, we have obtained an algorithm for construction of utility networks, which can be applied to modernize the existing networks, or to create new ones in accordance with the plan for development of modern cities. This method for constructing miscellaneous networks takes into account the features of network types at the stage of their development, as well as their hierarchy. In addition, the approximate algorithm proposed in the article allows us to construct the cheapest and at the same time reliable network object.

Keywords: distribution area; target products; consumers; linear facility; utility network; trace; primary network; secondary network; graph; hypernet; reliability; heuristics; alternative route.

\section{References}

1. Solovyev A. Yu. Algoritmy strukturnoi optimizatsii i metody analiza informatsionnykh protsessov $v$ sovremennykh telekommunikatsionnykh sistemakh: avtoref. dis.... kand. tekhn. nauk [Algorithms for Structural Optimization and Methods for Analysis of Information Processes in Modern Telecommunication Systems. Author's abstract of Cand. eng. sci. diss.]. Voronezh, 2011. $18 \mathrm{p}$.

2. Stennikov V. A., Chemezov A. A. Primenenie algoritma perebora derevyev i metoda imitatsii otzhiga dlya skhemno-strukturnoi optimizatsii teplovykh setei [Use of an Algorithm for Tree Search and an Annealing Simulation Method for Circuit-Based Optimization of Heat Networks]. Programmnye produkty i sistemy. 2018. No. 2 (31). Pp. 387-395.

3. Naumov I. V., Yamschikova I. V. Matematicheskoe obosnovanie vybora optimizatsionnoi modeli trassirovki elektricheskoi seti [Mathematical Substantiation of 
the Choice of the Optimization Model of Electrical Network Tracing]. Eurasian Union of Scientists (ESU). 2015. No. 7 (16). Pp. 123-127.

4. Stepanov V. P. Optimizatsiya marshrutov na dorozhnoi seti [Optimization of Routes on the Road Network]. Nauka i obrazovanie. 2012. No. 5. Pp. 1-12.

5. Popkov V. K., Toktoshov G. Y. Gipersetevaya tekhnologiya optimizatsii inzhenernykh setei v gornoi ili peresechennoi mestnosti [Hypernetwork Technology for Optimization of Utility Networks in Mountainous or Rough Areas]. Vestnik Buryatskogo gosudarstvennogo universiteta. Ser. Matematika i informatika. 2010. V. 9. Pp. 276-282.

6. Poulovassilis A., Levene M. A Nested-Graph Model for the Representation and Manipulation of Complex Objects. J. ACM Trans. Inf. Syst. 1994. V. 12. Pp. 35-68.

7. Kurant M., Thiran P. Layered Complex Networks. J. Phys. Rev. Lett. 2006. V. 96. Pp. 1-4.

8. Popkov V. K. O modelirovanii gorodskikh transportnykh sistem gipersetyami [About the Modeling of Urban Transport Systems by Hypernetworks]. Automation and Remote Control. 2011. V. 72. No. 6. Pp. 179-189.

9. Avtomatizirovannoe proektirovanie generalnogo plana sooruzhenii biokhimicheskoi ochistki stochnykh vod [Automated Structural Engineering for Biochemical Wastewater Treatment Facilities]. URL: http://www.gaps.tstu.ru/win1251/lab/gis/genplan.html (accessed 19.10.2018).

10. Rodionov A. S., Rodionova O. K. Random Hypernets in Reliability Analysis of Multilayer Networks. J. Lecture Notes in Electrical Engineering. 2015. V. 343. Pp. 307-315.

11. Rodionov A. S., Rodionova O. K. Using Random Hypernets for Reliability Analysis of Multilevel Networks. 1st Int. Conf. on Mathematical Methods and Computational Techniques in Science and Engineering (MMCTSE 2014). Ser. Mathematical Methods in Science and Engineering. Greece, Athens, 2014. Pp. 119-121.

12. Toktoshov G., Yurgenson A., Migov D. Design of Utility Network Subject to Reliability Constraint. Proc. of International Multi-Conference on Engineering, Computer and Information Sciences, IEEE SIBIRCON. Novosibirsk, 2017 (18-22 Sept. 2017). P. 172.

13. Toktoshov G. Y., Yurgenson A. N., Migov D. A. On a Problem of the Utility Network Design. OPTA-SCL 2018. Springer, 2018. V. 2098. Pp. 385-395.

14. Popkov V. K. Trudno reshaemye zadachi teorii gipersetei [Difficult Problems of the Theory of Hypernetworks]. Proc. of Russian Conf. on Discrete Optimization and Operations Research. Novosibirsk, 2007. Pp. 69-73.

15. Colbourn Ch. J. The Combinatorics of Network Reliability. New York: Oxford University Press, 1987. Pp. 143-157.

16. Popkov V. K., Toktoshov G. Y., Yurgenson A. N. Ob odnom podkhode k opti-mizatsii infrastruktury inzhenernykh setei [On a Certain Approach to Optimizing the Infrastructure of Utility Networks]. Vestnik SibGUTI. 2012. No. 3. Pp. 11-28.

17. Christofides N. Graph Theory. An Algorithmic Approach. New York: Academic Press, 1975. 